\title{
Enhancing plasmonic-photonic hybrid cavity modes by coupling of individual plasmonic nanoparticles
}

Shuaidi Zhang ${ }^{\dagger}$, Sajanlal R. Panikkanvalappils, Saewon Kang ${ }^{\dagger}$, Marcus J. Smith ${ }^{\dagger \#,}$ Shengtao $\mathrm{Yu}^{\dagger}$, Mostafa El-Sayed ${ }^{\S}$, Vladimir V. Tsukruk ${ }^{*}$

† School of Materials Science and Engineering, Georgia Institute of Technology, Atlanta, Georgia 30332, United States

$\S$ Laser Dynamics Laboratory, School of Chemistry and Biochemistry, Georgia Institute of Technology, Atlanta, Georgia 30332, United States

\# Aerospace Systems Directorate, Air Force Research Laboratory, Wright-Patterson Air Force Base, Dayton, Ohio 45433, United States
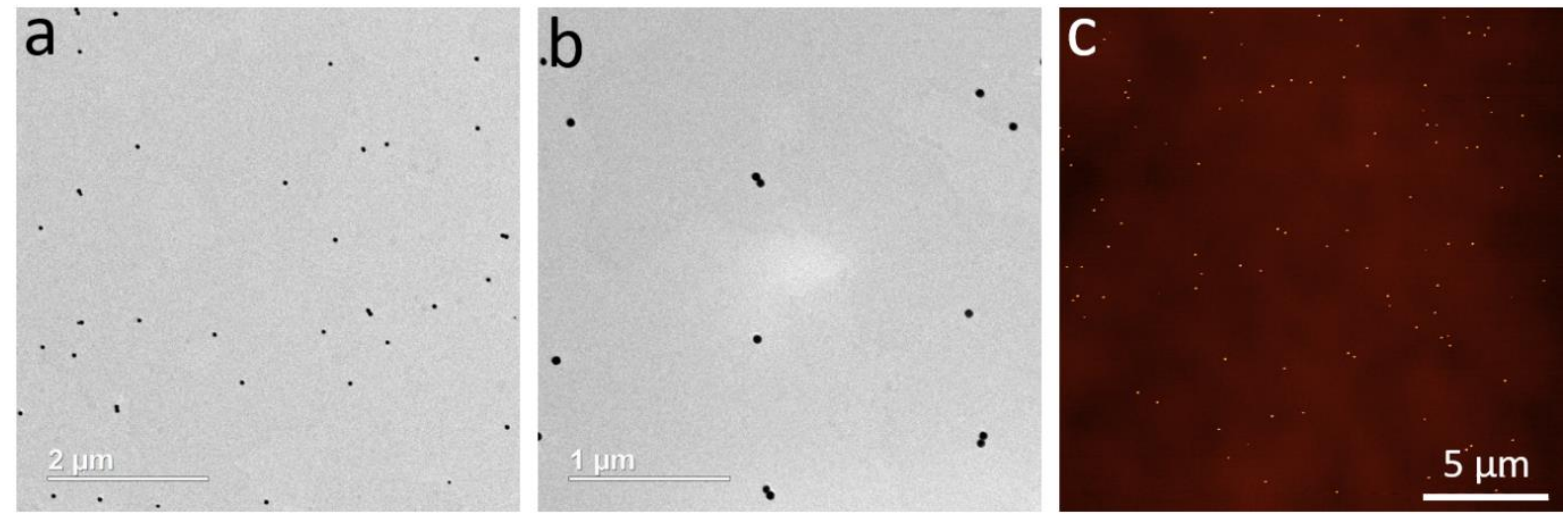

Figure S1. (a) Representative TEM image showing the AuNS to have a mean size of 52 $\mathrm{nm}$. (b) TEM image of larger magnification showing the presence of dimers among monomers. (c) Large area AFM topographical scan of AuNS spin-coated on FP cavity. Z scale: $70 \mathrm{~nm}$. 

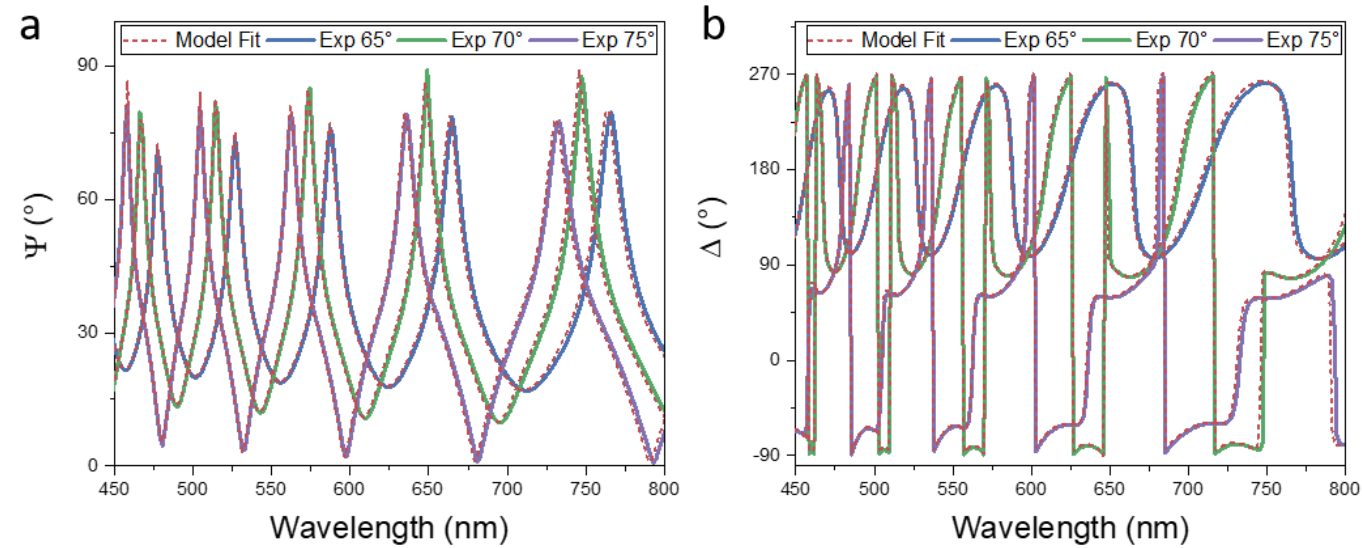

Figure S2. Spectroscopic ellipsometry measurement of thermally oxidized $\mathrm{SiO}_{2}$ wafer at $65^{\circ}$ (blue solid curve), $70^{\circ}$ (green solid curve), $75^{\circ}$ (purple solid curve) incident angles, and fit curve with $2170 \mathrm{~nm}$-thick $\mathrm{SiO}_{2}$ on $\mathrm{Si}$ model (red dashed curve) for $\Psi$ (a) and $\Delta$ (b) respectively.

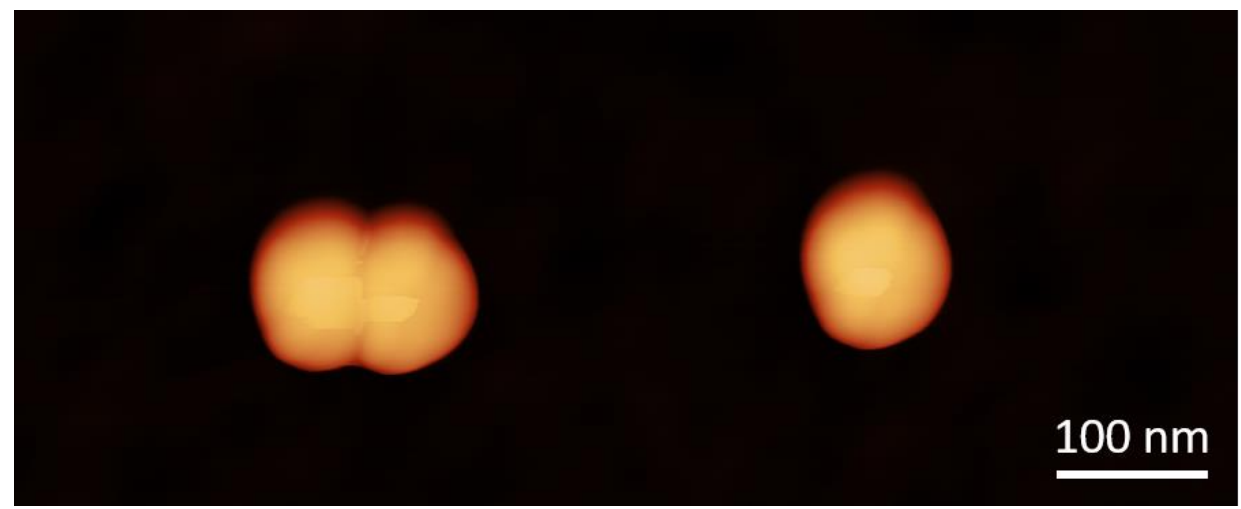

Figure S3. High resolution AFM topographical image of a dimer and a monomer in the same frame. $Z$ scale: $70 \mathrm{~nm}$ 


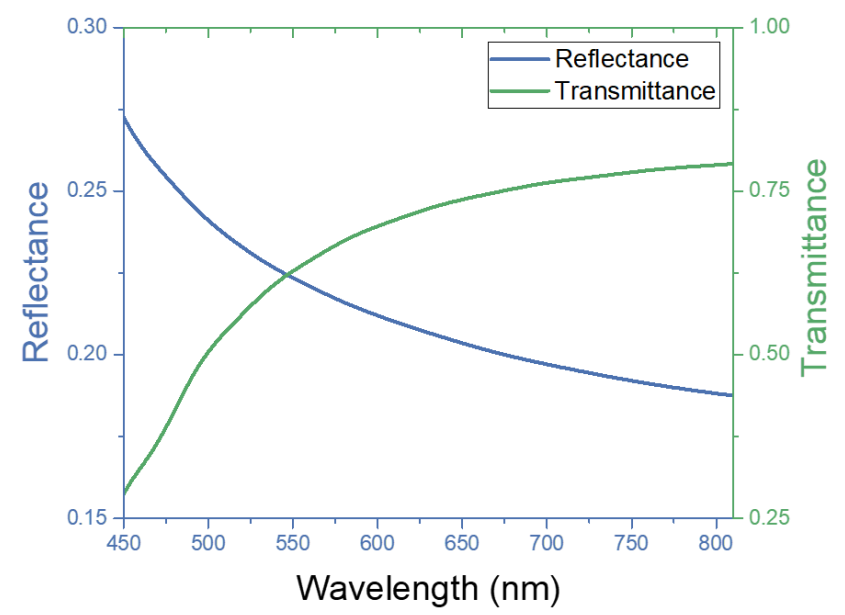

Figure S4. Calculated reflectance and transmittance of perfectly flat, semi-infinite, $\mathrm{SiO}_{2} / \mathrm{Si}$ interface (light going from $\mathrm{SiO}_{2}$ into $\mathrm{Si}$ )
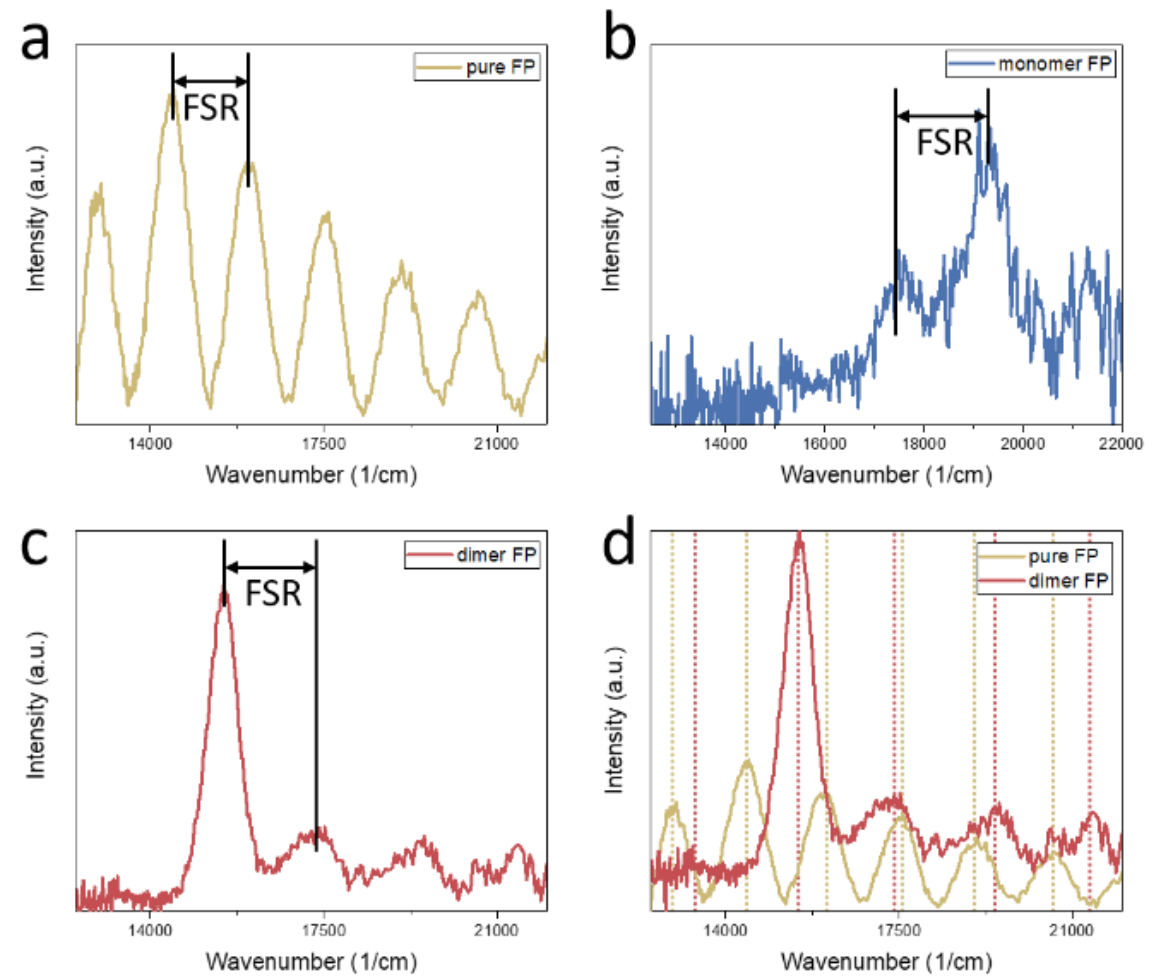

Figure S5. Spectra from Figure 4 converted to wavenumber space: (a) pure FP, (b) individual monomer on FP, (c) individual dimer on FP. (d) Superimposed spectra of dimer on FP and pure FP with vertical dashed lines of respective color marking their mode positions. 